\title{
العزة: تطبيق لوحة مفاتيح عربية ذكية للأجهزة المحمولة
}

\section{Arabic Izza: an Arabic smart app for mobile devices}

\section{أ. د. عبد المالك بوحجرة "Abdelmalek Bouhadjera}

لتسهيل الكتابة باللغة العربية باستخدام الأجهزة الذكية الحديثة (كالهواتف المحمولة و الألواح الإلكترونية...)، تم تطوير تطبيق "مفاتيح العزة الذكية" الذي يمكن تحميله على الرابط التالي: https://keyman.com/keyboards/arabic_izza وبعطي الثكل (1) صورة عامة عن هذا التطبيق.

$(\varphi)$

\begin{tabular}{|c|c|c|c|c|c|c|c|c|c|}
\hline 1 & 2 & 3 & 4 & 5 & 6 & 7 & 8 & 9 & 0 \\
\hline @ & $\begin{array}{c}\# \\
\text { shift } k_{3} 3\end{array}$ & $\%$ & $\begin{array}{c}* \\
\text { shifk } 6\end{array}$ & $=$ & 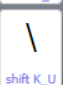 & $\div$ & $\begin{array}{c}\times \\
\text { shift } \mathrm{O}\end{array}$ & - & + \\
\hline$\sim$ & shift & I shift K_D & \{ & \} & shifk K. & ] & $<$ & $\stackrel{>}{>}$ & $\boldsymbol{x}$ \\
\hline$a b c$ & (7) & $\circ$ & $\mathrm{g}$ & & & & 5 & $\ddot{8}$ & هـ \\
\hline
\end{tabular}

\begin{tabular}{|c|c|c|c|c|c|c|c|}
\hline ص & ق & ف & $\varepsilon$ & 0 & ح & ج & ك \\
\hline س & ي & ب & I & ل & ت & ن & م \\
\hline ، & J & $s$ & ال & s & 9 & $b$ & د \\
\hline 123 & (7) & & & & 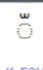 & $\leftrightarrow$ & $\boldsymbol{\theta}$ \\
\hline
\end{tabular}

شكل (1) تطبيق المفاتيح الأكية للكتابة بحروف عربية في الأجهزة الذكية (هواتف محمولة، ألواح ذكية...)

تُعد هذه المفاتيح إضافة مهمة لتطبيق مفاتيح العِزَّة المُستعمَل مع حواسيب المكتب 1، وهي تتناسب مع معظم أنظمة التشغيل الحالية. بالإضافة إلى احتواء هذا التطبيق على حروف الكتابة باللغة العربية وروزها، فهو يحتوي أيضاً على بعض الحروف والرموز المستعملة في لغات أخرى (كالأمازيغية والفارسية والكردية) التي تستخدم الحروف العربية، مع إمكانية إضافة رموز أخرى من لغات أخرى. وترتكز المفاتيح الذكية في هذا التطبيق على نفس المبادئ التي بُنيت عليها مفاتيح العِّة 1، لاسيما تمثيل الهززة برمز واحد مستقل، و اعتبارها كحرف أبجدي بحد ذاتها، كباقي الحروف. وكذلك، يركز هذا التطبيق على أهمية الحركات و الثدة بإظهارها في المستوى الأول من لوحة المفاتيح (دون الحاجة إلى الضغط على أكثر من مفتاح لإظهار الرمز المطلوب)، مما يسهل كثيراً من عملية إدخال النصوص العربية المُشَكََّّة، و دراسة العربية و تعلّمها.

الأستاذ الدكتور عبد المالك بوحجرة، الجزائر، مر اسلة إلى: Prof Dr. Abdelmalek Bouhadjera, Algeria, Email: bouhadjeraa@yahoo.com 
وفي التطبيق المخصص للألواح الإلكترونية، تم اعتماد توزيع المفاتيح كما هي في حواسيب المكتب، مع وجود رموز التتقبط على المستوى الأول دون الحاجة إلى استخدام المستوى الثاني. أما في التطبيق المخصص للهواتف المحمولة، فهناك ثمانية رموز في كل سطر في المستوى الأول (شكل 1. أ)، حيث تظهر الأزرار في مربعات منتابهة، مما يوفر لها مساحة أكبر و وضوح أكثر.

أما الحروف التي لا تَظهر على المستوى الأول، فيمكن الوصول إليها كما يلي:

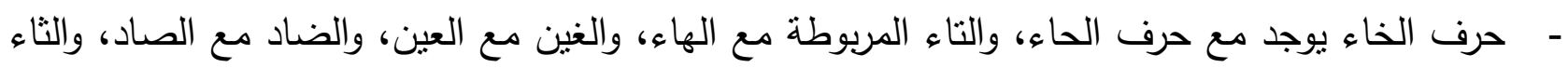

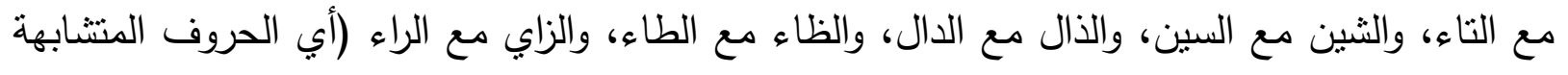
بعضها مع بعض). - لكتابة الألف المهوزة (أ)، بالفتح أو الضم، يُضْنغط على حرف الألف مرتين متتاليتين، وحرف الألف بهمزة سفلية (إ) بالضغط على زر الهمزة مرتين، والهمزة على واو (ؤ) بالضغط على حرف الواف الواو مرتين، والهمزة

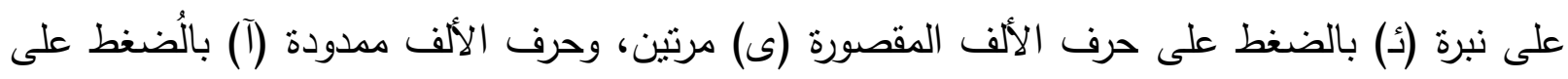
الهمزة ثم الألف. - ألف التعريف (ال): لها زِرّ مستقل كما في مفاتيح الحاسب المكتبي. - علامات التشكيل (الفتحة والضمة والكسرة) توجد مع الثدة.

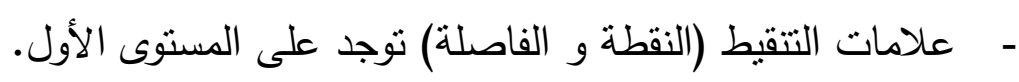

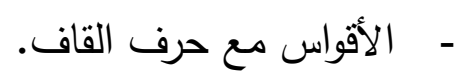

- الرموز الجديدة (ج، ث، گ، ب، زّ) توجد مع الحروف العربية المشابهة لها تباعاً (ج، ف، لك، ب، ز ). وإلى جانب تلك الخصائص، هناك إمكانية لإضافة رموز بلغات أخرى كالتركية التي كانت تعتمد الأحرف العربية سابقاً.

مراجع

1. Bouhadjera, A., (2018). مفاتيح العزة: لوحة مفاتيح عربية لتسهيل كتابة وتعلم العربية في عصر المعلوماتية. Arabic Science Archive (arabixiv): https://doi.org/10.17605/osf.io/p2y38 